\title{
The Continuous Spectrum of Time-Harmonic Shear Layers
}

\author{
M.J. Philipp Hack and Tamer A. Zaki \\ Department of Mechanical Engineering \\ Imperial College London \\ London $S W^{r}$ 2AZ
}

\begin{abstract}
In boundary layers, eigenfunctions which belong to the continuous spectrum of the Orr-Sommerfeld equation have been established as a suitable basis for the expansion of general free-stream vortical disturbances. They are oscillatory in the free stream, and attenuate inside the boundary layer due to shear sheltering. The extent of modal penetration into the shear depends on the disturbance frequency and orientation, with the low-frequency, streamwise elongated modes being the most effective triggers of a high-amplitude streak-like response. The influence of introducing a time-periodic, spanwise mean flow on modal sheltering is investigated. The evaluation of the continuous modes in this case requires a Floquet expansion in the fundamental frequency of the base flow. Appropriate treatment of the free-stream boundedness condition is developed, and quantitative measures of modal sheltering are computed. The time-dependent, spanwise motion is shown to significantly enhance shear sheltering, and to change the optimal orientation of the continuous modes for penetration into the shear. An explanation is provided, in the limit of low frequency of the base flow, using asymptotic analyses.
\end{abstract}




\section{INTRODUCTION}

In boundary layers, eigensolutions which belong to the continuous branch of the OrrSommerfeld spectrum represent free-stream vorticity modes. The eigenfunctions have therefore been established as a suitable basis for the expansion of free-stream vortical perturbations. An inviscid mechanism, known as "shear sheltering", generally restricts the ability of these vortical modes to penetrate the mean shear. However, the effectiveness of shear sheltering is not uniform: Earlier studies have demonstrated that low-frequency modes can effectively penetrate the shear, and lead to the amplification of boundary layer streaks which precede bypass transition to turbulence. The characteristics of these modes, in particular their structure in the near-wall region, take a pivotal role in flow stability and are consequently of relevance for a range of applications in aeronautics and engineering. The present work investigates the effect of the introduction of time-dependent shear. In addition to the steady Blasius profile, the base flow includes a time-harmonic spanwise component defined by the second problem of Stokes. The objective is to clarify the impact of unsteady shear on the sheltering of free-stream vortical modes.

Jordinson [1] conjectured that the discrete Orr-Sommerfeld eigensolutions, in the case of semi-infinite domains, are supplemented by a continuous spectrum. The latter is nevertheless exponentially stable, and was therefore irrelevant to the classical linear stability analysis of the Tollmien-Schlichting mechanism. Mack [2] was able to show the equivalence of the phase speed of the continuous modes and the free-stream convective velocity in the limit of large Reynolds numbers. The same work numerically demonstrated the sinusoidal behavior of continuous modes in the free stream, in contrast to discrete modes which vanish away from the wall. The formal mathematical description of the continuous spectrum was provided in Grosch and Salwen [3]. The authors suggested that even though the expansion of arbitrary disturbances requires a full eigenbasis consisting of both the discrete and the continuous 
part of the spectrum, arbitrary perturbations at large wall distances may be represented by a superposition of continuous modes only. Jacobs and Durbin [4] advanced this hypothesis by defining broadband, free-stream turbulence as in terms of a superposition of continuous modes.

Inviscid rapid distortion theory predicts that free-stream vortical disturbances do not cause a perturbation inside the mean shear - they are expelled [5, 6]. This behavior was related by Craik [7] to an inviscid mechanism, active in the presence of mean shear and significantly more effective than viscous dissipation. The terminology of shear sheltering was coined later by Hunt and Durbin [8] when they discovered the pertinence of inviscid filtering for the interaction of vortices of significantly varying length scales. Quantitative studies of free-stream vortical mode sheltering in Blasius boundary layers have been performed by Jacobs and Durbin [4]. The authors found the penetration of vortical modes into the boundary layer to be inversely proportional to both the Reynolds number and modal frequency. A detailed study of sheltering, and the structure of the vortical modes in single- and two-fluid shear, was performed by Zaki and Saha [9]. They employed asymptotic as well as numerical approaches in order to comprehensively explain the underlying mechanism. The effect of three-dimensionality of the base flow - as found along swept wings - on the continuous modes was examined by Schrader et al. [10]. They reported a significant increase in modal sheltering compared with two-dimensional boundary layers.

Investigations of the continuous spectrum, and the structure of vortical modes in boundary layers, are to material extent motivated by the ability of free-stream disturbances to initiate bypass transition. The latter refers to subcritical breakdown to turbulence, significantly upstream of transition via discrete instability waves. Jacobs and Durbin [11] performed direct numerical simulations of the bypass process, which they described as "somewhere between turbulence and transition", due to its stochastic nature. They synthesized isotropic free-stream turbulence at the inlet of their computational domain via weighted superposition of a spectrum of continuous Orr-Sommerfeld modes. The same approach was later used by Brandt et al. [12] in order to study the influence of the free-stream turbulent length scale on subcritical breakdown. In order to differentiate the role of the low- and highfrequency components of the free-stream vortical spectrum, Zaki and Durbin [13] carried out simulations of bi-modal interactions, leading to breakdown to turbulence. The latter work demonstrated that low-frequency, Orr-Sommerfeld modes lead to the generation of 
Klebanoff streaks. The high-frequency modes are sheltered, and hence are only observed in the free stream. However, their interaction with the streaks, near the edge of the boundary layer, leads to secondary instability and breakdown to turbulence.

The base flows in the above studies, both in two- or three-dimensional boundary layers, were steady. The literature on the effects of unsteady mean motion has generally focused on fully-turbulent, wall-bounded shear flows [14-16]. However, relatively less attention was attributed to the effect of wall-oscillation on transitional flows, and in particular the interaction of free-stream vortical disturbances with boundary layers. The recent work by Ricco [17] demonstrated that superposing a streamwise-dependent, spanwise mean flow onto a boundary layer can lead to weaker streaks. However, the base flow was steady, and the freestream disturbances which were considered belonged to a narrow parameter range. Other vortical modes could potentially yield an enhanced response. A systematic study of the influence of unsteady shear on the proceedings of bypass transition - namely the penetration of vortical modes into the boundary layer, the amplification of streaks and nonlinear breakdown to turbulence - is therefore not available in the literature. The first of these questions is addressed herein.

The present work examines the sheltering properties of streamwise boundary layers when a time-periodic, spanwise mean motion is introduced. In $\S 2$, the eigenvalue problem which governs linear perturbations to the base state is introduced. A solution method in terms of a finite order Floquet expansion is described, as formally introduced by Hall [18] and Herbert [19]. In addition, a method for computing the continuous modes, and enforcing a boundedness condition in the free stream, is presented within the context of the Floquet expansion. In $\S 3$, quantitative measures of modal sheltering are defined and evaluated for different frequencies and amplitudes of the base flow oscillation. Finally, the deductions based on the numerical solutions of the eigenvalue problem are augmented by asymptotic analyses in $\S 4$, in the limit of low frequency mean-flow oscillation.

\section{THEORETICAL FORMULATION}

An illustration of the base flow considered herein is given in figure 1. It is a superposition of a streamwise Blasius boundary-layer $U(y)$, and a time-periodic spanwise velocity component $W(y, t)$. The latter is modelled by the solution to the familiar second problem 


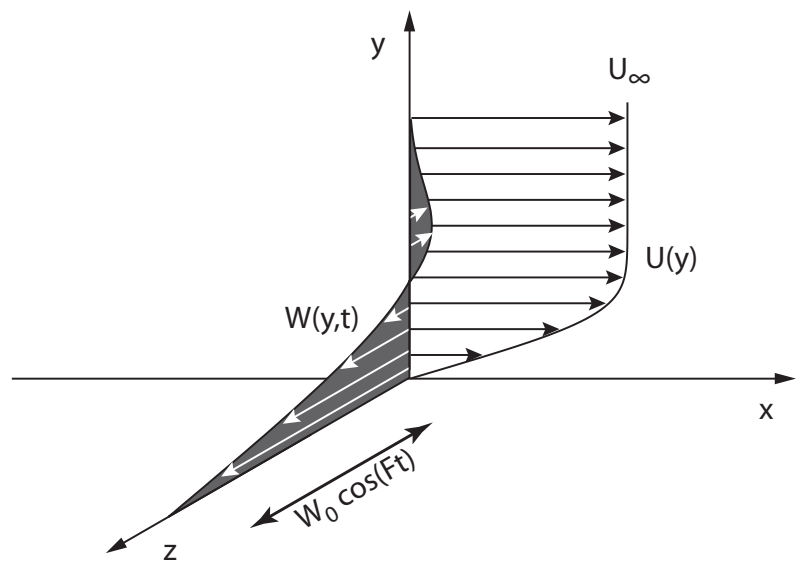

FIG. 1: Illustration of the base flow, $\underline{U}=(U, 0, W(y, t))^{T}$

of Stokes,

$$
W(y, t)=W_{0} \exp \left(-\sqrt{\frac{R e \pi}{T}} y\right) \cos \left(\frac{2 \pi}{T} t-\sqrt{\frac{R e \pi}{T}} y\right),
$$

where $W_{0}$ is the magnitude of the wall oscillation velocity, $T$ is the period of wall oscillation. The Reynolds number, $R e \equiv U_{\infty} \delta / \nu$, is based on the Blasius flow, where $\delta$ is the $99 \%$ boundary layer thickness and $U_{\infty}$ is the Blasius free-stream speed. Throughout this work, lengths are scaled by $\delta$, velocities are made non-dimensional using $U_{\infty}$, and a value $R e=1000$ is used.

In the presence of the streamwise flow, the Stokes relation (1) is no longer an exact solution to the Navier-Stokes equations. Direct numerical simulations nevertheless show that the relative error in this ansatz is less than one percent for the flow parameters of interest (see Appendix A). Sufficiently large Reynolds numbers further justify the application of a parallel-flow approximation. The full base flow is therefore,

$$
\underline{U}=(U(y), 0, W(y, t))^{T} .
$$

\section{A. Governing equations for linear perturbations}

The linear evolution equations for a small perturbation, $\underline{u}^{\prime}=\left(u^{\prime}, v^{\prime}, w^{\prime}\right)^{T}$, to the base state are derived starting from the Navier-Stokes equations for the full velocity, $\underline{U}+\underline{u^{\prime}}$. The equations for the base flow are subtracted, and nonlinear terms in $\underline{u}^{\prime}$ are neglected. An 
equation of Orr-Sommerfeld type is obtained for the wall-normal perturbation component,

$$
\left[\left(\frac{\partial}{\partial t}+U \frac{\partial}{\partial x}+W \frac{\partial}{\partial z}\right) \nabla^{2}-\mathcal{D}^{2} U \frac{\partial}{\partial x}-\mathcal{D}^{2} W \frac{\partial}{\partial z}-\frac{1}{R e} \nabla^{4}\right] v^{\prime}=0
$$

where $\mathcal{D} \equiv \partial / \partial y$. The coefficients $U, W, \mathcal{D}^{2} U$ and $\mathcal{D}^{2} W$ are independent of the wallparallel $x$ and spanwise $z$ dimensions. Under the assumption of wave-like disturbances in these directions, equation (2) thus admits solutions of the form

$$
v^{\prime}(x, y, z, t)=v(y, t) \exp \left(\mathrm{i} k_{x} x\right) \exp \left(\mathrm{i} k_{z} z\right)
$$

The temporal problem is considered here, so that the streamwise and spanwise disturbance wavenumbers $k_{x}$ and $k_{z}$ are known, real quantities. Strict time periodicity of $W$ and $\mathcal{D}^{2} W$ requires a Floquet expansion of the perturbation, in the fundamental frequency $F=2 \pi / T$ of the base flow,

$$
v(y, t)=\exp (\sigma t) \sum_{n=-\infty}^{\infty} v_{n}(y) \exp (\mathrm{i} n F t) .
$$

The exponent $\sigma=\sigma_{\mathrm{r}}+\mathrm{i} \sigma_{\mathrm{i}}$ is the complex frequency. Following established notation, the real part $\sigma_{\mathrm{r}}$ gives the disturbance growth rate, whilst the imaginary part $\sigma_{\mathrm{i}}$ denotes a frequency shift from integer multiples of $F$.

The complete ansatz for the disturbance velocity (3) is substituted in the governing equation (2), and yields the differential equation,

$$
\begin{array}{r}
\sum_{n=-\infty}^{\infty}\left[\left(-\mathrm{i} \sigma+n F+k_{x} U+k_{z} W\right)\left(\mathcal{D}^{2}-\kappa^{2}\right)-k_{x} \mathcal{D}^{2} U\right. \\
\left.-k_{z} \mathcal{D}^{2} W+\mathrm{i} \frac{1}{R e}\left(\mathcal{D}^{2}-\kappa^{2}\right)^{2}\right] \exp (\mathrm{i}(-\sigma+n F) t) v_{n}(y)=0,
\end{array}
$$

where $\kappa^{2}=k_{x}^{2}+k_{z}^{2}$. For a given wavenumber pair $\left(k_{x}, k_{z}\right)$, only particular values of $\sigma$ admit solutions of the system (5), which gives rise to an eigenvalue problem in $\sigma$. The corresponding eigenfunctions $v$ are recovered from the harmonic eigen-components $v_{n}$ as given in equation (4).

By taking into account the periodicity of the base flow, equation (5) can be recast as a set of coupled ordinary differential equations. First, the spanwise base flow (1) is expressed in the form,

$$
W(y, t)=W_{1}(\exp (\mathrm{i} F t)+\exp (-\mathrm{i} F t))+W_{2}(\exp (\mathrm{i} F t)-\exp (-\mathrm{i} F t))
$$


where $W_{1}=W_{0} \exp (a y) \cos (a y) / 2, W_{2}=\mathrm{i} W_{0} \exp (a y) \sin (a y) / 2$ and $a=-\sqrt{F R e / 2}$. Substituting the above expression in the eigenvalue problem (5), and grouping terms that premultiply $\exp (\mathrm{i} F \mathrm{Ft})$, we obtain the set of coupled ordinary differential equations,

$$
\begin{aligned}
& {\left[\left(-\mathrm{i} \sigma+n F+k_{x} U\right)\left(\mathcal{D}^{2}-\kappa^{2}\right)-k_{x} \mathcal{D}^{2} U+\mathrm{i} \frac{1}{R e}\left(\mathcal{D}^{2}-\kappa^{2}\right)^{2}\right] v_{n}(y)} \\
& +k_{z}\left(W_{1}+W_{2}\right)\left(\mathcal{D}^{2}-\kappa^{2}-2 \mathrm{i} a^{2}\right) v_{n-1}(y) \\
& +k_{z}\left(W_{1}-W_{2}\right)\left(\mathcal{D}^{2}-\kappa^{2}+2 \mathrm{i} a^{2}\right) v_{n+1}(y)=0 .
\end{aligned}
$$

In the final form, each Floquet component, $v_{n}$, is coupled to $v_{n \pm 1}$ due to the time-harmonic base flow.

\section{B. The continuous spectrum}

The dispersion relation of the continuous Orr-Sommerfeld spectrum is derived by considering the free-stream limit of the system of governing equations (5). As $y \rightarrow \infty, U=U_{\infty}$ and $W, \mathcal{D}^{2} U, \mathcal{D}^{2} W=0$, and as such all time-dependent coefficients vanish. The Floquet shape functions $v_{n}(y)$ hence become decoupled in the free stream, and must each satisfy the relation,

$$
\left[\left(-\mathrm{i} \sigma+n F+k_{x} U\right)\left(\mathcal{D}^{2}-\kappa^{2}\right)+\mathrm{i} \frac{1}{R e}\left(\mathcal{D}^{2}-\kappa^{2}\right)^{2}\right] v_{n}^{\infty}=0 .
$$

Note that the eigenvalue $\sigma$ is unchanged among all the Floquet components, (i.e. $\sigma$ is the same for all values of $n$ ). However, in order to determine $\sigma$, one must consider a particular, although arbitrary, choice of $n=\widetilde{n}$. The Floquet mode, $v_{n=\widetilde{n}}^{\infty}$, is nontrivial. All other components $v_{n \neq \tilde{n}}^{\infty}$ must vanish in the free stream in order to satisfy (7). They become finite inside the Stokes layer due to coupling between Floquet modes via the periodic base state.

With the particular choice, $n=\widetilde{n}$, equation (7) becomes,

$$
\left[\left(-\mathrm{i} \sigma+\widetilde{n} F+k_{x} U\right)\left(\mathcal{D}^{2}-\kappa^{2}\right)+\mathrm{i} \frac{1}{R e}\left(\mathcal{D}^{2}-\kappa^{2}\right)^{2}\right] v_{\widetilde{n}}^{\infty}=0 .
$$

Solutions to this equation are of the form $v_{\widetilde{n}}^{\infty}(y)=\sum_{j=1}^{4} C_{j} \exp \left(\lambda_{j} y\right)$, where $\lambda_{j}$ is the $j$-th root of the characteristic polynomial of equation (8),

$$
\begin{aligned}
& \lambda_{1,2}^{2}=\kappa^{2} \\
& \lambda_{3,4}^{2}=\kappa^{2}+\mathrm{i} R e\left(-\mathrm{i} \sigma+\widetilde{n} F+k_{x} U_{\infty}\right) .
\end{aligned}
$$


One of the first two solutions, $\lambda_{1,2}$, must be discarded since it is exponentially growing with distance from the wall and is therefore unphysical. If $\lambda_{3,4}$ are complex, a similar argument requires elimination of the growing component. As a result, the eigenfunction, $v_{\widetilde{n}}^{\infty}(y)=C_{1} \exp \left(\lambda_{1} y\right)+C_{3} \exp \left(\lambda_{3} y\right)$, vanishes in the free stream. However, the two degrees of freedom can not satisfy the four boundary conditions associated with the fourth order differential equation; this gives rise to the discrete spectrum of the Orr-Sommerfeld operator.

Alternatively, the continuous spectrum is obtained by requiring that the eigenfunctions remain bounded, rather than vanish, in the free stream. For boundedness, purely imaginary values of $\lambda_{3,4}$ are sought by setting $\lambda_{3,4}^{2}=-k_{y}^{2}$. The free-stream behavior of these modes is therefore proportional to $\exp \left( \pm \mathrm{i} k_{y} y\right)$, where $k_{y}$ is a real parameter that governs the wallnormal wavenumber of the continuous-spectrum modes. The dispersion relation for the continuous spectrum is therefore,

$$
\sigma=-\mathrm{i}\left(k_{x} U_{\infty}+\widetilde{n} F\right)-\frac{1}{R e}\left(k_{x}^{2}+k_{y}^{2}+k_{z}^{2}\right) .
$$

The real component of the eigenvalue, $\sigma_{\mathrm{r}}$, is a continuous function of the parameter, $k_{y}$, and hence the terminology "continuous spectrum". It should also be noted that the dispersion relation reduces to the expression for Blasius flow when the base state is steady, $F=0$. When $F$ is finite, the Floquet nature of the problem introduces multiple continuous branches, separated by a frequency shift, $F$. Each branch corresponds to a particular choice of $\widetilde{n}$.

A graphical representation of the eigenvalue spectrum is obtained from a numerical solution of the Floquet problem, and is shown in figure 2. Because of the discrete nature of the computational method, continuous branches are approximated by a finite set of eigenvalues. Each vertical line in the figure is a continuous branch, with constant frequency $\sigma_{\mathrm{i}}$ obtained from a particular choice of $\tilde{n}$.

The choice of $\widetilde{n}$ and the wavenumber vector $\underline{k}=\left(k_{x}, k_{y}, k_{z}\right)^{T}$ fully defines an eigenvalue. The corresponding eigenfunction remains a superposition of the full Floquet expansion (4), and solution to the eigenvalue problem (5). Thence, four boundary conditions are required for each component of the Floquet expansion. At the wall, they must each satisfy,

$$
\begin{gathered}
v_{n}(y=0)=0, \\
\mathcal{D} v_{n}(y=0)=0 \forall n .
\end{gathered}
$$

In the free stream, since the eigenvalue $\sigma$ was derived from boundedness of $v_{\widetilde{n}}^{\infty}$, the boundary conditions for this particular Floquet component must reflect such behavior. Boundedness 


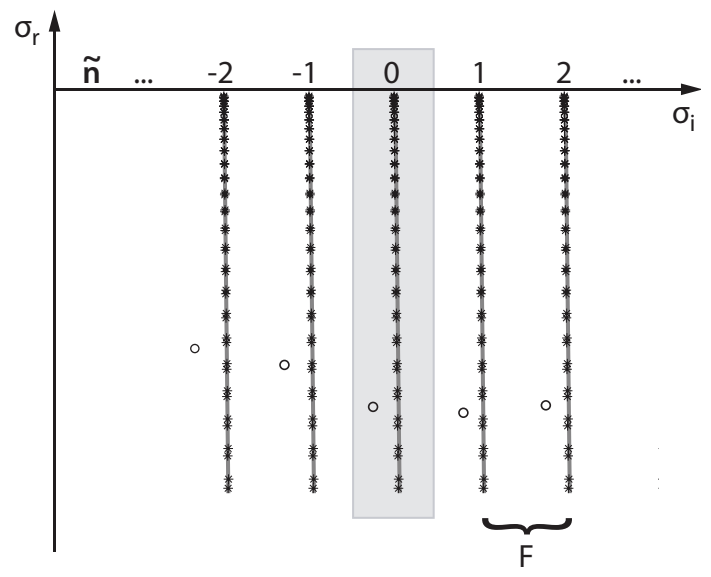

FIG. 2: Schematic of the Floquet spectrum: ॰, discrete eigenvalues; *, eigenvalues of the continuous branches.

is enforced using the approach proposed by Jacobs and Durbin [4],

$$
\begin{aligned}
v_{\tilde{n}}^{\infty} & =1, \\
\frac{\left(\mathcal{D}^{2} v_{\tilde{n}}^{\infty}+k_{y}^{2} v_{\tilde{n}}^{\infty}\right)_{y 1}}{\left(\mathcal{D}^{2} v_{\widetilde{n}}^{\infty}+k_{y}^{2} v_{\widetilde{n}}^{\infty}\right)_{y 2}} & =\exp \left(k_{y}\left(y_{2}-y_{1}\right)\right),
\end{aligned}
$$

where $y_{1}$ and $y_{2}$ are two points in the free stream. As stated above, the remaining Floquet components, $v_{n \neq \tilde{n}}^{\infty}$ must vanish in the free stream. This can be shown formally: given an eigenvalue $\sigma(\widetilde{n})$ from the dispersion relation (9), equation (7) is satisfied by Floquet components with $n \neq \widetilde{n}$ if and only if $v_{n \neq \tilde{n}}^{\infty}$ vanishes,

$$
\begin{aligned}
v_{n}^{\infty} & =0, \\
\mathcal{D} v_{n}^{\infty} & =0, n \neq \widetilde{n} .
\end{aligned}
$$

\section{Numerical Method}

Numerical solution of the eigenvalue problem (5) requires a finite Floquet expansion, $-N \leq n \leq N$. The resulting generalized eigenvalue problem has the form $A \underline{v}=\sigma B \underline{v}$. Since the eigenvalues of the continuous spectrum are known a priori from the dispersion relation (9), the eigen-problem can be converted into a boundary value problem described by a system of linear equations,

$$
(A-\sigma B) \underline{v}=\underline{b},
$$


where the vector $\underline{v}$ is a combined representation of all $2 N+1$ harmonic shape functions $v_{n}(y)$. Nontrivial solutions require an inhomogeneous right-hand side with at least one nonzero element in $\underline{b}$. The latter is given by the free-stream boundedness of the $\widetilde{n}$-th harmonic component $v_{\widetilde{n}}$, as described in equations (12) and (13). Although $\widetilde{n}$ is arbitrary, a value of $\widetilde{n}=0$ had been employed throughout this work. This choice identifies the continuous branch which coincides with the continuous spectrum of the Blasius base flow - see equation (9).

In order to solve the boundary value problem (16), a similar approach to that of Liu et al. [20] and Vaughan and Zaki [21] is used. A spectral Chebyshev discretization is applied in the wall-normal coordinate. An algebraic coordinate mapping is used in order to ensure that half of the Gauss-Labatto points are located inside the boundary layer. Numerical experiments established that 200 Chebyshev polynomials, and a domain height of fifty boundary layer thicknesses, $y \in[0,50 \delta]$, are sufficient for an accurate representation of the eigenfunction $v$. A direct factorization of $(A-\sigma B)$ into a lower and an upper triangle operator is employed in order to solve equation (16) for the continuous mode, $\underline{v}$.

A simple validation of the above described Floquet approach for computing continuous modes is given for the case of a pure Blasius boundary layer. In this limit of steady base flow, all time-dependent coefficients of the governing equation (2) vanish. The modal behavior must consequently be described by a single term of the Floquet expansion, such that the ansatz (4) may be equivalently replaced by a normal mode assumption with respect to time, $v^{\prime}(y, t)=v(y) \exp (\sigma t)$. An example comparison of the Floquet solution and the normal mode assumption is given in figure 3, where both solutions are shown to match identically.

When the base flow is periodic, all components $v_{n}$ of the Floquet expansion are coupled and contribute to the makeup of the eigenfunction, as shown in figure 4. The contributions of the zeroth $(\widetilde{n})$ and first harmonic $(\widetilde{n} \pm 1)$ terms are of the same order of magnitude in the boundary layer. All Floquet components with $n \neq \tilde{n}$ vanish in the free stream, where the amplitude of the time-periodic base flow becomes negligible. Independent of the considered wall distance, higher Floquet components fail to significantly contribute to the total mode shape. Numerical studies in this work thus employ Floquet expansions to the tenth harmonic of the fundamental frequency. This component was in none of the considered cases found to be of material magnitude. 


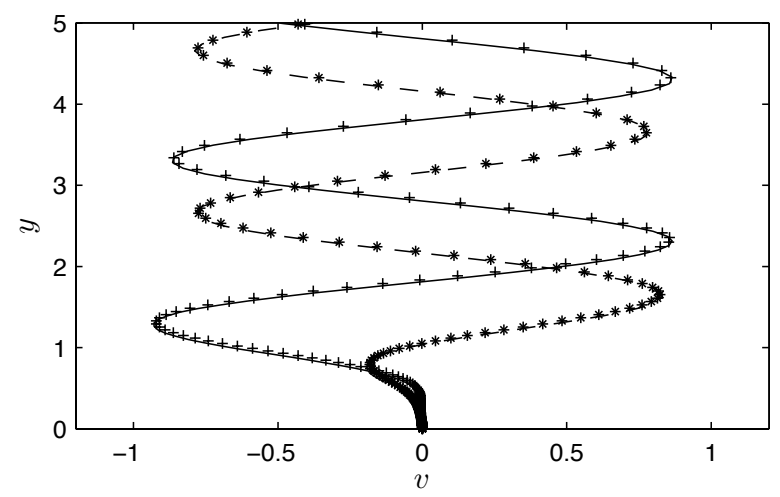

FIG. 3: Comparison of the continuous modes computed using the normal mode and Floquet ansatz for Blasius base flow. The modal wavenumber vector, $\underline{k}=(0.25, \pi, \pi)^{T} \cdot(+)$ real and $(*)$ imaginary components of the normal modes; (_- ) real and (- - ) imaginary component of the Floquet solution.
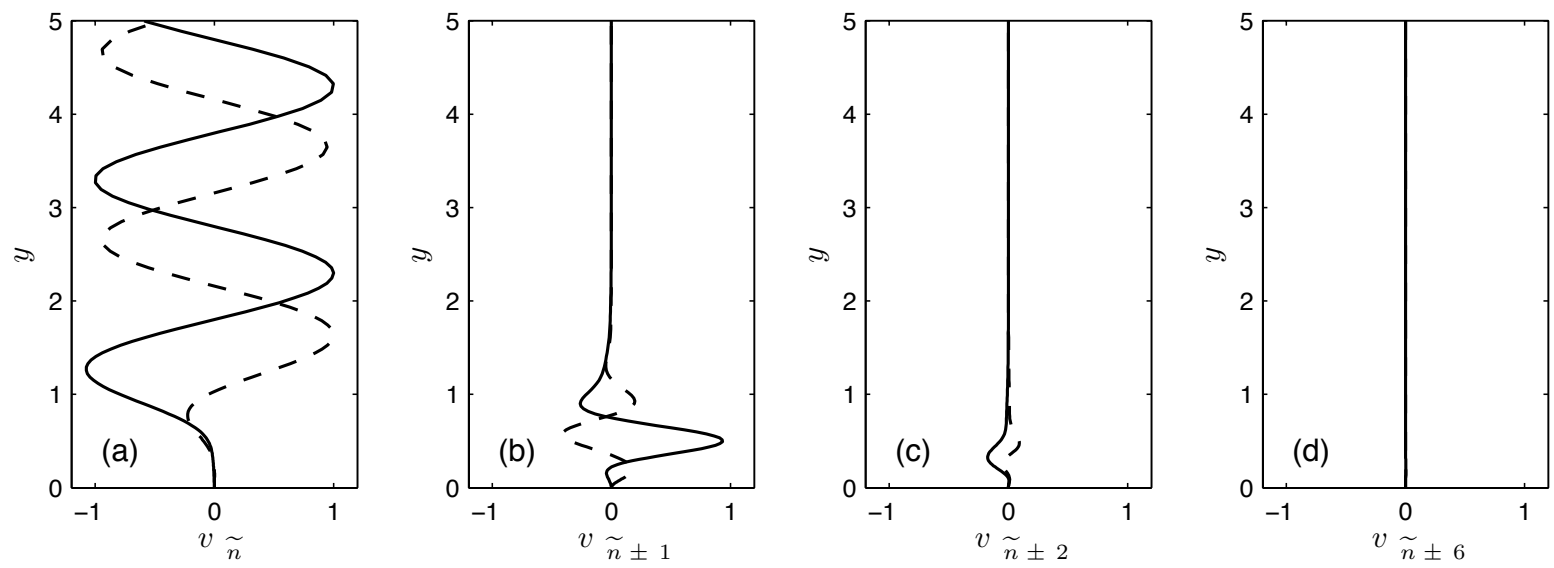

FIG. 4: Selected harmonic solution components $v_{n}$ of the Floquet expansion for $T=40$, $W_{0}=0.25 U_{\infty} ;$ (a) $n=\widetilde{n}$; (b) $n=\widetilde{n} \pm 1$; (c) $n=\widetilde{n} \pm 2$; (d) $n=\widetilde{n} \pm 6$; — real component; - - -, imaginary component

\section{RESULTS}

\section{A. Modal Sheltering}

Streamwise oriented vortical disturbances play a significant role in the context of subcritical transition to turbulence in boundary layers. Andersson et al. [22] demonstrated 
that streaks emanating from streamwise aligned vortices yield the largest algebraic energy growth, but the initial perturbation must be located within the boundary layer. Jacobs and Durbin [4] investigated the ability of mean shear to shelter the boundary layer from free-stream vortical disturbances. They demonstrated that the penetration of free-stream modes into a Blasius layer is proportional to the the streamwise disturbance wavelength. Streamwise-elongated vortical modes are therefore the highest amplified and weakest sheltered vortical perturbations of Blasius layers. In accordance with these earlier findings, figure 5 (a) demonstrates the very weak sheltering of a vortical perturbation of wavenumber vector $\underline{k}=(0.01, \pi, \pi)^{T}$ in pure Blasius flow. Introduction of an unsteady Stokes layer substantially changes the sheltering characteristics, as is shown in figures $5(\mathrm{~b}-\mathrm{d})$. A reduction of modal penetration with increasing base flow period is observed, corresponding to a growing thickness of the spanwise Stokes layer $d_{\text {Stokes }} \sim \sqrt{T}$. In figures $5(\mathrm{~b}-\mathrm{c})$, the ratio $d_{\text {Stokes }} / \delta<1$. On the other hand, in figure $5(\mathrm{~d})$, the thickness of the Stokes layer exceeds that of the Blasius flow, $d_{\text {Stokes }} / \delta=1.64$. This results in the enhanced sheltering observed in the mode shape in that case.
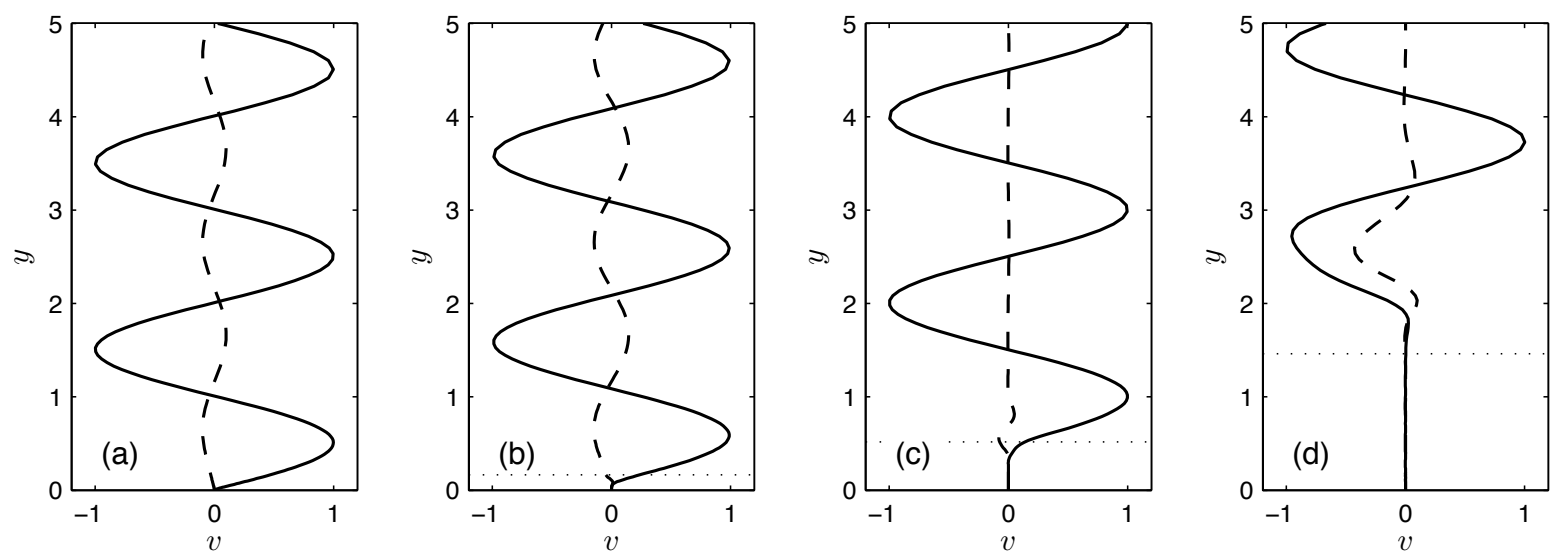

FIG. 5: Modal sheltering for (a) Blasius and $(\mathrm{b}-\mathrm{d})$ Stokes-Blasius flow. The dotted line marks the thickness of the Stokes layer. $W_{0}=0.25 U_{\infty}, T=\{4,40,400\}, d_{\text {Stokes }} / \delta=\{0.16,0.52,1.64\}$; (—) real and (- - - ) imaginary components of the eigenfunction.

Since spanwise wall oscillation reorients the mean shear, it is expected that the timedependent mean flow will exhibit different sheltering characteristics from the Blasius counterpart. The effectiveness of the base flow to shelter arbitrarily oriented free-stream vortical 
modes is quantified by introducing the integral parameter,

$$
s=\int_{0}^{\delta} \frac{v_{\infty}-v(y)}{v_{\infty}} \mathrm{d} y
$$

where $v_{\infty}$ is the average free-stream amplitude of the eigenfunction. This quantity approaches unity for high sheltering with vanishing modal penetration into the boundary layer. Weakly damped free-stream modes, which retain their full amplitude throughout the boundary layer correspond to small values of $s$. Throughout this work, the wall-normal disturbance wavelength is kept on the order of the boundary layer thickness, $2 \pi / k_{y} \sim \mathcal{O}(1)$. This particular choice also found in other works on vortical interactions with shear layers is motivated by the observation of strong blocking for low- $k_{y}$ modes. On the other hand, eigenfunctions with high wall-normal wavenumber have a high viscous decay rate, and due to their small amplitude do not induce an appreciable response inside the shear layer [23].

A numerical evaluation of the sheltering parameter $s$ for a steady boundary layer and a wide range of wavenumber pairs, $\left(k_{x}, k_{z}\right)$, is shown in figure 6. For pure Blasius flow, modal permeability into the shear is nearly independent of the spanwise wavenumber. A decrease in the streamwise wavenumber thus directly corresponds to increased modal amplitude within the shear layer. Presence of unsteady spanwise motion substantially changes the sheltering characteristics (see figure 7). In contrast to the case of steady one-dimensional flow, the level of sheltering now additionally depends on the spanwise wavenumber of the eigenmode. Free-stream vortical modes with relatively high $k_{z}$ are sheltered. For smaller wave numbers, the permeability of the shear layer depends on the ratio of streamwise and spanwise wavenumbers, $k_{x} / k_{z}$. The origin of this dependence is elucidated in section IV, where it is related to the orientation of the mean shear.

\section{B. Energy Sheltering}

Incomplete sheltering of free-stream vortical disturbances results in a fraction of the free-stream turbulent kinetic energy being introduced into the boundary layer. This energy may serve as an initial seed for the algebraic growth mechanism that ultimately leads to subcritical breakdown. In the following, a von Kármán spectrum is assumed in the free stream, and an integral measure for the total disturbance energy within the boundary layer is derived. The influence of wall oscillation on the energy inside the shear is also evaluated 


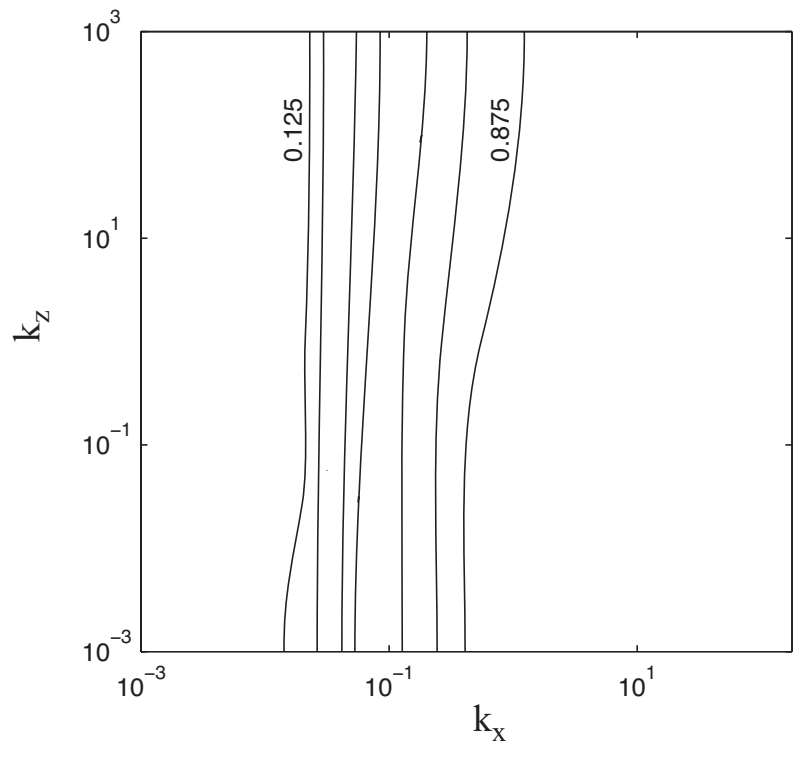

FIG. 6: Quantitative evaluation of modal sheltering for Blasius flow; lines denote levels from 0.125 to 0.875 with an increment of 0.125

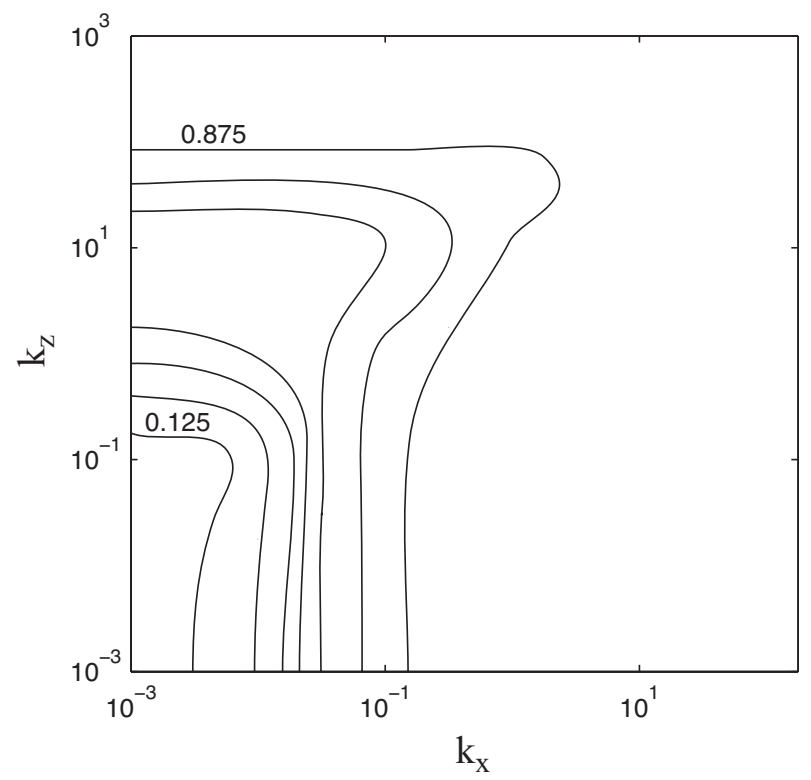

FIG. 7: Quantitative evaluation of modal sheltering for combined Stokes-Blasius flow of $T=100$, $W_{0}=0.25 U_{\infty} ;$ lines denote levels from 0.125 to 0.875 with an increment of 0.125

for a range of base-flow frequencies and amplitudes.

Eigenfunctions of the continuous Orr-Sommerfeld and Squire spectra behave like Fourier 
modes in the free stream,

$$
\begin{aligned}
& v^{\prime} \sim \hat{v} \exp \left(\mathrm{i}\left(k_{x} x+k_{y} y+k_{z} z\right)\right), \\
& \eta^{\prime} \sim \hat{\eta} \exp \left(\mathrm{i}\left(k_{x} x+k_{y} y+k_{z} z\right)\right),
\end{aligned}
$$

where $\eta \equiv \partial u / \partial z-\partial w / \partial x$ is the wall-normal vorticity. The continuous spectrum modes therefore constitute a suitable basis for the expansion of a broadband perturbation field [11]. The scaling $\hat{v}=-\mathrm{i} A \sqrt{k_{x}^{2}+k_{z}^{2}} / k, \hat{\eta}=-\mathrm{i} B \sqrt{k_{x}^{2}+k_{z}^{2}}$ leads to the Cartesian formulation,

$$
\begin{aligned}
& \hat{u}=\frac{\mathrm{i} A k_{x} k_{y}}{k \sqrt{k_{x}^{2}+k_{z}^{2}}}+\frac{B k_{z}}{k_{x}^{2}+k_{z}^{2}}, \\
& \hat{v}=-\mathrm{i} \frac{A \sqrt{k_{x}^{2}+k_{z}^{2}}}{k}, \\
& \hat{w}=\frac{\mathrm{i} k_{y} k_{z} A}{k \sqrt{k_{x}^{2}+k_{z}^{2}}}-\frac{B k_{x}}{k_{x}^{2}+k_{z}^{2}},
\end{aligned}
$$

where $k \equiv \sqrt{k_{x}^{2}+k_{y}^{2}+k_{z}^{2}}$. Isotropy of the disturbance field enables disregard of all directional information, so that a scalar formulation of the energy spectrum is obtained. Von Kármán [24] proposed the specific relation,

$$
E(k)=\frac{L^{5} k^{4}}{C\left(1+(k L)^{2}\right)^{17 / 6}},
$$

which behaves as $E(k) \sim k^{4}$ for large scales, and recovers the property $E(k) \sim k^{-5 / 3}$ in the inertial sub-range as implied by the second similarity hypothesis of Kolmogorov. Following Jacobs and Durbin [11], we set $L=\frac{55 C}{9 \pi} L_{11}$ and $C=0.6884$. A unit integral turbulent lengthscale, $L_{11}=1$, leads to a peak in the energy spectrum at unit wavenumber. In addition, the total energy contained within the spectrum is $\int_{0}^{\infty} E(k) \mathrm{d} k=3 / 2$.

The kinetic energy within the wavenumber range $\left[\underline{k}_{1}, \underline{k}_{2}\right]$ can be expressed in terms of $\mathcal{F}^{2}(k) \equiv E(k) /\left(2 \pi k^{2}\right)$, according to,

$$
\epsilon=\int_{k_{z 1}}^{k_{z 2}} \int_{k_{y 1}}^{k_{y 2}} \int_{k_{x 1}}^{k_{x 2}} \frac{1}{2} \mathcal{F}^{2}(k) \mathrm{d} k_{x} \mathrm{~d} k_{y} \mathrm{~d} k_{z} .
$$

Alternatively, $\epsilon$ can also be evaluated from the coefficients of the Fourier expansion (17),

$$
\begin{aligned}
\epsilon & =\int_{k_{z 1}}^{k_{z 2}} \int_{k_{y 1}}^{k_{y 2}} \int_{k_{x 1}}^{k_{x 2}} \frac{1}{2}\left(\hat{u} \hat{u}^{*}+\hat{v} \hat{v}^{*}+\hat{w} \hat{w}^{*}\right) \mathrm{d} k_{x} \mathrm{~d} k_{y} \mathrm{~d} k_{z} \\
& =\int_{k_{z 1}}^{k_{z 2}} \int_{k_{y 1}}^{k_{y 2}} \int_{k_{x 1}}^{k_{x 2}} \frac{1}{2}\left(A A^{*}+B B^{*}\right) \mathrm{d} k_{x} \mathrm{~d} k_{y} \mathrm{~d} k_{z},
\end{aligned}
$$


where star denotes the complex conjugate. Equating the two expressions for $\epsilon$, and assuming isotropy of the disturbance field, leads to the particular choice,

$$
\begin{aligned}
& A=\mathcal{F} \exp \left(\mathrm{i} \theta_{1}\right) \cos \gamma \\
& B=\mathcal{F} \exp \left(\mathrm{i} \theta_{2}\right) \sin \gamma,
\end{aligned}
$$

where $\theta_{1}, \theta_{2}$ and $\gamma$ are uniformly distributed random angles [25]. In this context, $\gamma$ governs the energy distribution between the Orr-Sommerfeld and Squire modes, whilst $\theta_{j}$ specifies the spatial phase of the perturbations.

It follows from the above that the spectral energy density due to the wall-normal perturbation $v(\underline{k})$ alone is

$$
\begin{aligned}
\rho_{v}(\underline{k}) & =\frac{1}{2} \hat{v}(\underline{k}) \hat{v}^{*}(\underline{k}) \\
& =\frac{1}{2} \frac{E(k)}{4 \pi k^{2}} \frac{k_{x}^{2}+k_{z}^{2}}{k^{2}} .
\end{aligned}
$$

Since the random angle $\gamma$ is uniformly distributed, the $\cos ^{2}(\gamma)$ term has been substituted by its arithmetic mean of one half. Integration of $\rho_{v}(\underline{k})$ over the three-dimensional wavenumber space $\underline{k}$ gives one third of the total energy of all perturbation components, $\epsilon_{v}=\int_{0}^{\infty} \int_{0}^{\infty} \int_{0}^{\infty} \rho_{v}(\underline{k}) \mathrm{d} k_{x} \mathrm{~d} k_{y} \mathrm{~d} k_{z}=1 / 2$.

In the following, attention is focused on the energy spectrum near $\widetilde{k}_{y}=\pi$. These modes, whose wall-normal scale is on the order of one boundary layer thickness penetrate the shear most effectively and undergo maximum energy amplification. The total kinetic energy of $\widetilde{k}_{y}$ modes within the boundary layer can be evaluated from the integral,

$$
\epsilon_{v \widetilde{k}_{y}}=\frac{1}{\delta} \int_{0}^{\delta} \int_{k_{x}} \int_{k_{z}} \rho_{v}(\underline{k})\|v(y ; \underline{k})\| \mathrm{d} k_{z} \mathrm{~d} k_{x} \mathrm{~d} y
$$

where $v(y ; \underline{k})$ is the eigenfunction of the Orr-Sommerfeld continuous spectrum. Normalisation by the disturbance energy inside the boundary layer for pure Blasius base flow yields the

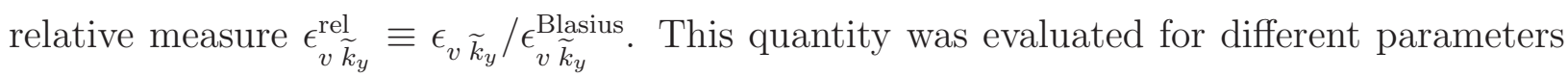
of the spanwise base flow, and is shown in figure 8. The fraction of free-stream energy which penetrates the boundary layer is inferred to decrease with increasing period and amplitude of the wall oscillation.

Previous examination of the sheltering measure $s$ in $\S$ III A has shown a shift in the modal wavenumber, or equivalently modal orientation, for which the weakest sheltering is observed. 


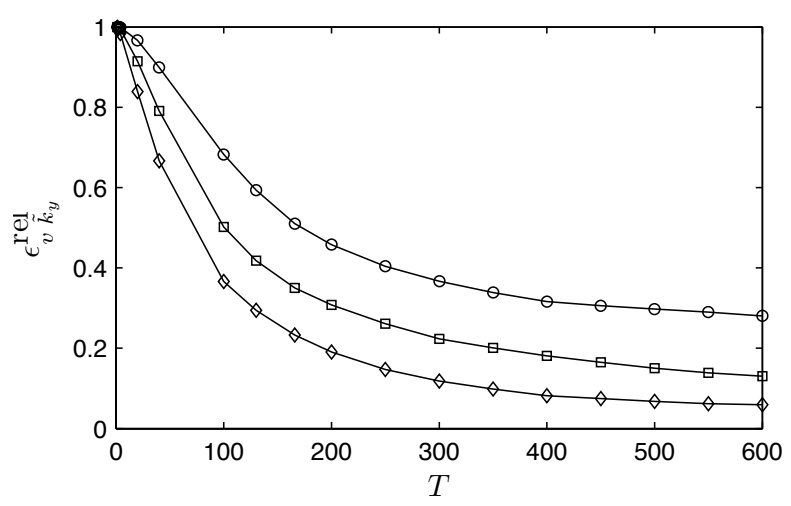

FIG. 8: Normalized von Kármán-weighted modal energy within the boundary layer for $k_{y}=\pi$; , $W_{0}=0.1 U_{\infty} ; \square, W_{0}=0.2 U_{\infty} ; \diamond, W_{0}=0.4 U_{\infty}$

This motivates a comparison of the perturbation energy within the boundary layer along rays of constant $k_{z} / k_{x}$, which define modes oriented at an angle $\varphi=\tan ^{-1}\left(k_{z} / k_{x}\right)$ relative to the streamwise direction. Integrating the energy spectrum along lines of constant ratio $k_{z} / k_{x}$ yields,

$$
\begin{aligned}
& \epsilon_{v \widetilde{k}_{y}}^{\varphi}=\frac{1}{\epsilon_{v \widetilde{k}_{y}}^{\text {Blasius }}} \frac{1}{\delta} \int_{0}^{\delta} \int_{k_{x}} \int_{k_{z}} \rho_{v}(\underline{k})\|v(y ; \underline{k})\| \mathrm{d} k_{z} \mathrm{~d} k_{x} \mathrm{~d} y, \\
& \text { with } k_{z}=k_{x} \tan \varphi .
\end{aligned}
$$

Integration over all angles recovers the total energy of the $v$-perturbation spectrum within the boundary layer, $\int_{0}^{\pi / 2} \epsilon_{v \widetilde{k}_{y}}^{\varphi} \mathrm{d} \varphi=\epsilon_{v \widetilde{k}_{y}}^{\mathrm{rel}}$. Figure 9 shows the variation of $\epsilon_{v \widetilde{k}_{y}}^{\varphi}$ with the angle $\varphi$. An increase in the amplitude of the base-flow oscillation causes a shift of the maximum energy towards higher values of $\varphi$. In addition, modes oriented at very large angles to the streamwise direction insignificantly contribute to the total energy.

The angle $\varphi_{\epsilon}$ max which maximizes the perturbation energy inside the boundary layer is charted in figure 10, for a range of base-flow oscillation frequencies and amplitudes. For Blasius flow, it is known that $\varphi_{\epsilon \max }=0$, or that streamwise oriented modes are most penetrating into the boundary layer and thus contribute most to the disturbance energy inside the shear. As the period and magnitude of wall oscillation are increased, $\varphi_{\epsilon} \max$ shifts away from zero. The maximum contribution to the energy spectrum in the boundary layer is therefore due to oblique vortical modes.

The presence of spanwise unsteady shear hence not only reduces the magnitude of bound- 


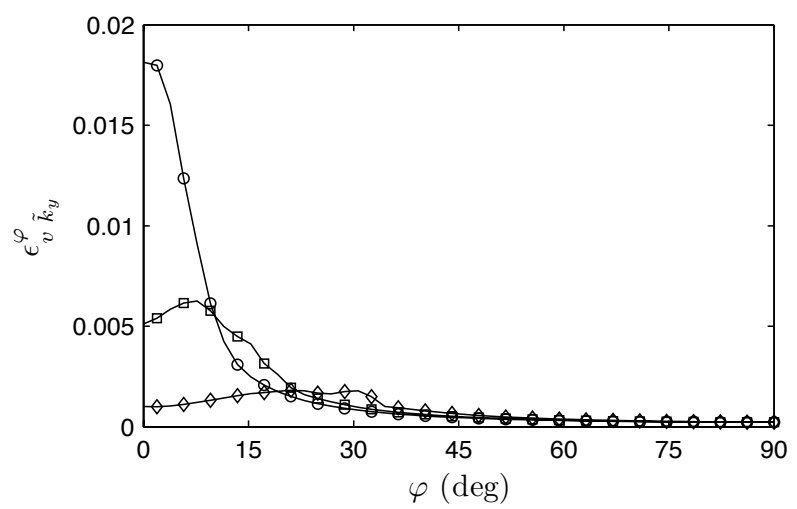

FIG. 9: Angular distribution of weighted modal energy for $k_{y}=\pi$ and $T=300 ; \circ, W_{0}=0.1 U_{\infty}$; $\square, W_{0}=0.2 U_{\infty} ; \diamond, W_{0}=0.4 U_{\infty}$

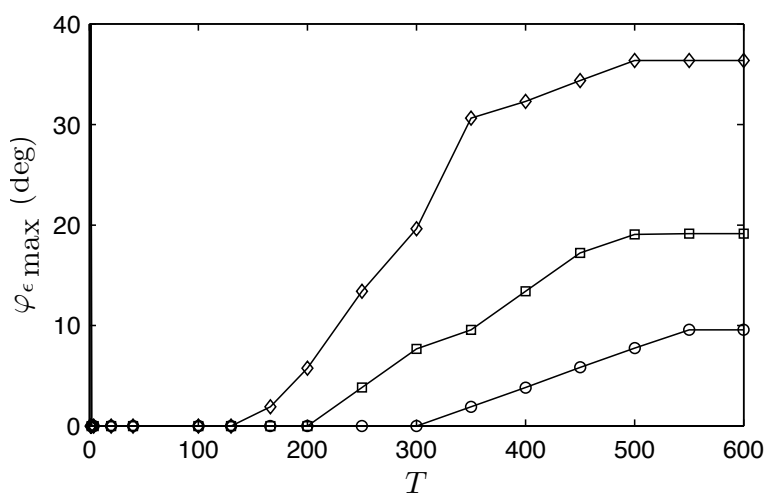

FIG. 10: The direction of weakest disturbance energy sheltering for $k_{y}=\pi ; \circ, W_{0}=0.1 U_{\infty} ; \square$, $W_{0}=0.2 U_{\infty} ; \diamond, W_{0}=0.4 U_{\infty}$

ary layer perturbation energy, but also changes the modal parameters for which the weakest sheltering is observed. Significantly increased disturbance damping inside the shear is observed for streamwise elongated perturbations. Depending on the parameters of the spanwise base flow, modes aligned in an angle to the streamwise direction may on the other hand be subject to decreased sheltering.

\section{Timescale-based model of shear sheltering}

A physical interpretation of shear sheltering in Blasius boundary layers was provided by Zaki and Saha [9]. They explained the sheltering phenomenon in terms of convective and 
diffusive processes: The convection of the free-stream vortical disturbance relative to an observer within the shear introduces a convective timescale. In addition, according to the Orr-Sommerfeld equation, the free-stream vorticity may only reach the observer by wallnormal diffusion which itself carries an inherent timescale. When the convective timescale is short, diffusion processes can not act sufficiently fast and the observer is sheltered from the free-stream disturbance; such is the case for high-frequency continuous modes and at high relative convection speed due to strong shear. Based on this physical interpretation, a model of shear sheltering in combined Stokes-Blasius flow is formulated and numerically evaluated in this section.

The phase speed of the continuous modes is equal to the free-stream mean-flow velocity. These modes therefore appear steady in a Lagrangian reference frame, or relative to an observer, that translates at $U_{\infty}$. On the other hand, a fluid particle within the shear travels at a lower streamwise velocity, and hence experiences a temporal change of the modal phase. The apparent frequency $\Omega_{x}$, as recorded by the observer, is therefore equal to the product of the relative convection speed and the streamwise disturbance wavenumber,

$$
\Omega_{x}=\left(U_{\infty}-U(y)\right) k_{x}
$$

At the wall, one recovers the frequency in the lab frame, which is the temporal frequency from the modal dispersion relation (9), $\Omega_{x}=U_{\infty} k_{x}=\omega_{\mathrm{r}}$.

Combined Stokes-Blasius flow introduces an additional spanwise relative motion between the free-stream mode and locations within the shear layer. The apparent frequency, $\Omega_{x, z}$, due to the combined streamwise and spanwise relative convection is thus,

$$
\Omega_{x, z}=\left(U_{\infty}-U(y)\right) k_{x}+W_{\mathrm{rms}}(y) k_{z}
$$

Based on this apparent frequency, the convective timescale is defined to be $T_{\mathrm{c}}=\Omega_{x, z}^{-1}$.

Due to the vanishing wall-normal mean velocity, $\underline{U}=(U(y), 0, W(y, t))^{T}$, free-stream vortical modes of the Orr-Sommerfeld equation (2) enter the boundary layer by wall-normal diffusion. Thence transport of modal information in the wall-normal direction is governed by the time scale,

$$
T_{\mathrm{d}} \sim \frac{R e}{k_{y}^{2}}
$$

which is a function of Reynolds number and wall-normal wavenumber. Following Zaki and Saha [9], the degree of sheltering can be determined by comparing the convective and 
diffusive time scales,

$$
\mathcal{N} \equiv \frac{T_{\mathrm{d}}}{T_{\mathrm{c}}}=\frac{\operatorname{Re}\left(k_{x}\left(U_{\infty}-U(y)\right)+k_{z} W_{\mathrm{rms}}(y)\right)}{k_{y}^{2}} .
$$

As $\mathcal{N} \rightarrow 0$, the time scale at which the wall-normal diffusion process is able to transport phase information is small compared to the convective time scale associated with the relative modal convection. Locations within the Stokes layer thus receive an exact representation of the modal phase information as illustrated in figure 11 (a). High values of the parameter $\mathcal{N} \rightarrow \infty$, on the other hand, correspond to a temporal under-resolution of the convective time scale by the diffusive mechanism (see figure 11 (b)). The observer within the shear thus receives a smeared representation of modal phase information, or is "sheltered" from the free-stream mode. It should be noted that the extremal cases of pure Stokes and pure Blasius flow are contained in the above definition of $\mathcal{N}$ via $U(y)=0$ and $W_{\text {rms }}=0$, respectively.

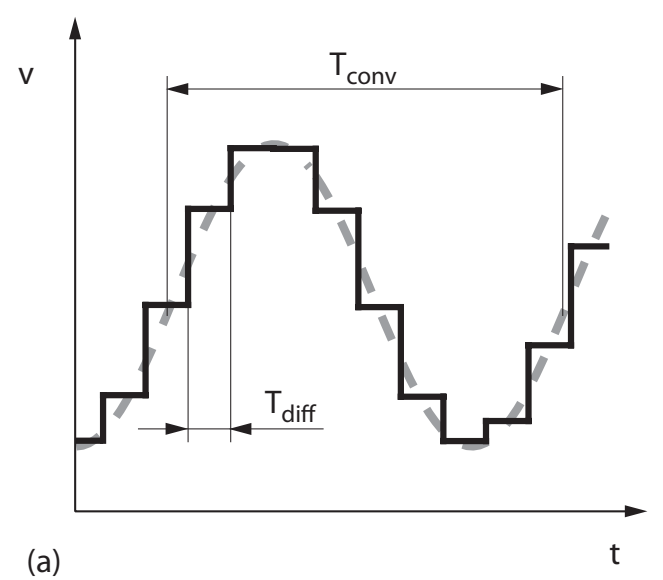

FIG. 11: Relevant time scales of modal sheltering observed in a Lagrangian frame of reference within the shear layer

Jacobs and Durbin [4] quantified sheltering by evaluating the distance from the edge of a Blasius boundary layer to the location where the vortical mode has attenuated to a fraction of its free-stream amplitude. In the current work, the spanwise wall movement introduces a second shear layer whose thickness depends on the frequency of oscillation. Therefore, it is more natural to measure modal penetration in terms of the height from the wall, where the vortical mode amplitude reaches one percent of its free-stream value,

$$
D_{1} \equiv \min _{y}:\|v(y)\|=0.01\left\|v_{\mathrm{FS}}\right\|
$$


Making use of the above model, a direct correlation between the wall distance $D_{1}$ and the parameter $\mathcal{N}$ is sought, such that

$$
\tilde{\mathcal{N}}=\frac{\operatorname{Re}\left(k_{x}\left(U_{\infty}-U\left(y=D_{1}\right)\right)+k_{z} W_{\text {rms }}\left(y=D_{1}\right)\right)}{k_{y}^{2}}=\text { const. }
$$

A numerical evaluation of this relation has been performed for a range of oscillation amplitudes $W_{0} \in[0.1,0.4] U_{\infty}$, periods $T \in[10,100]$ and streamwise and spanwise wavenumbers $k_{x}, k_{z} \in\left[10^{-3}, 10^{2}\right]$. The wall-normal disturbance length scales are selected to be of the order of the boundary layer thickness, $k_{y}=\{\pi, 2 \pi\}$. Figure 12 shows a comparison of $D_{1}$ extracted from the mode shapes following $(26)$ and the predicted value $D_{1}(\tilde{\mathcal{N}})$ from equation (27) with $\widetilde{\mathcal{N}}=6$. The agreement demonstrates that the time-scale based model introduced above yields a good approximation of modal sheltering in the presence of time-harmonic shear.

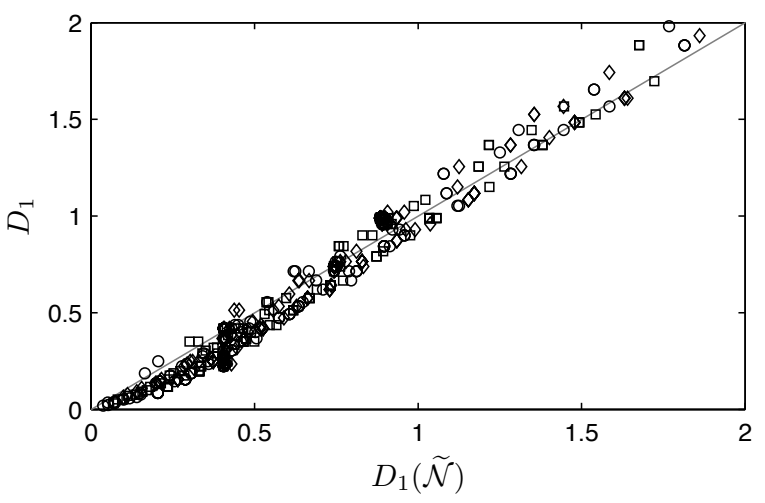

FIG. 12: Correlation of predicted and observed $D_{1} ; \circ, W_{0}=0.1 U_{\infty} ; \square, W_{0}=0.2 U_{\infty} ; \diamond, W_{0}=$ $0.4 U_{\infty}$

\section{ASYMPTOTIC ANALYSIS}

In order to explain the structure of the continuous modes in the presence of mean shear, Zaki and Saha [9] performed asymptotic analyses of the Orr-Sommerfeld equation for steady boundary layers. Analytical treatment of the problem was made possible by approximating the Blasius profile with a piecewise linear velocity distribution. Based on the magnitude of the parameter

$$
\mathcal{R} \equiv \frac{k_{x} U_{\infty} R e}{k_{y}^{2}}
$$


three distinct regimes were identified:

1. $\mathcal{R} \ll 1$ is the diffusive regime, where modes retain their free-stream amplitude throughout the largest portion of the boundary layer;

2. $\mathcal{R} \gg 1$ is the convective-sheltered regime, where modes are expelled from the boundary layer and are simply convected in the free stream;

3. $\mathcal{R} \sim \mathcal{O}(1)$ is an intermediate, "convective-diffusive" regime which exhibits limited modal penetration into the outer region of the boundary layer.

In order to explain the sheltering properties of combined Blasius-Stokes flow, an asymptotic approach is adopted, similar to the work of Zaki and Saha [9]. The analysis assumes a piecewise linear velocity profile for both the streamwise and the spanwise velocity component. The limit of large oscillation periods $T \gg \delta / U_{\infty}$ is considered, which allows a quasi-steady base flow assumption. Furthermore, the analysis focuses on cases where the Stokes and Blasius boundary layer thicknesses are comparable, $d \sim \mathcal{O}(\delta)$, and therefore $T \sim \mathcal{O}\left(\operatorname{Re} \delta^{2} \pi\right) \gg \delta / U_{\infty}$. A schematic of the base flow is shown in figure 13 , in a frame convecting with the free-stream speed, $U_{\infty}$. In this frame, the base-flow profile is given according to,

$$
\begin{array}{rlrl}
\mathcal{U}(Y) & =0 & & \text { for } Y>0, \\
\mathcal{U}(Y) & =\frac{U_{\infty}}{\delta} Y & \text { for } 0 \geq Y \geq-\delta, \\
\mathcal{W}\left(Y_{\mathrm{s}}\right)=0 & \text { for } Y_{\mathrm{s}}>0, \\
\mathcal{W}\left(Y_{\mathrm{s}}\right)=\frac{-W_{0}}{d} Y_{\mathrm{s}} & \text { for } 0 \geq Y_{\mathrm{s}} \geq-d,
\end{array}
$$

where $\mathcal{U} \equiv U-U_{\infty}$, and the wall-normal coordinates are $Y \equiv y-\delta$ and $Y_{\mathrm{s}} \equiv y-d$. Without loss of generality, the spanwise mean velocity is assumed to be contained within the streamwise boundary layer, $d<\delta$. Therefore, in the moving frame, the mean flow consists of a one-dimensional outer layer and a two-dimensional inner layer. The former was addressed in the work of Zaki and Saha [9], and the analysis herein will focus on the latter.

The governing equation for a small disturbance $v^{\prime}=v(y) \exp \left(\mathrm{i}\left(k_{x} x+k_{z} z-\omega t\right)\right)$ is obtained by introducing the above base flow, and the dispersion relation of the continuous modes, into the Orr-Sommerfeld equation (2). In terms of $\psi \equiv\left(\mathcal{D}^{2}-\kappa^{2}\right) v$, the disturbance 


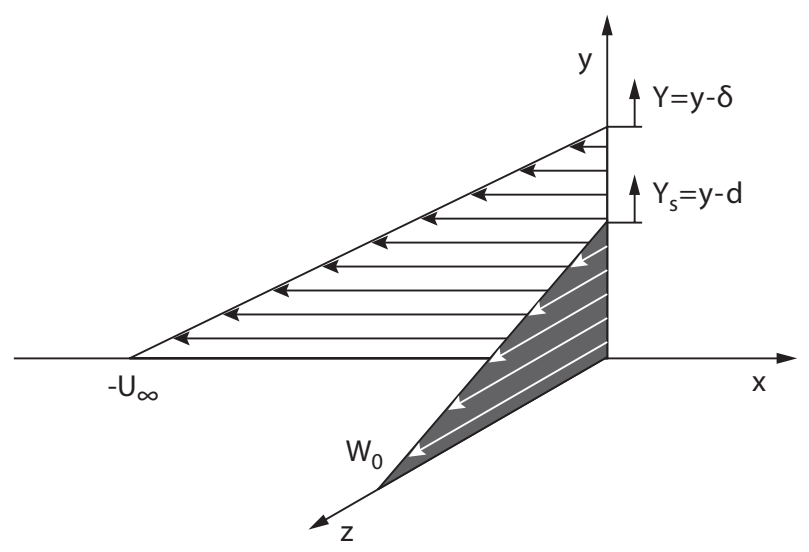

FIG. 13: Two-dimensional shear layer in the streamwise moving reference frame

equation is given by,

$$
\left[-\mathrm{i}\left(k_{x} \mathcal{U}+k_{z} \mathcal{W}\right)-1 / \operatorname{Re}\left(k_{y}^{2}+\mathcal{D}^{2}\right)\right] \psi=0
$$

Modes that are significantly damped by the outer layer are not of interest. Instead, the focus is on modes which belong to the diffusive regime of that region, $\mathcal{R} \equiv k_{x} U_{\infty} R e / k_{y}^{2} \ll 1$. These modes are unaffected by the outer shear, and are governed by the following equation for $Y_{\mathrm{s}}>0$,

$$
\mathcal{D}^{2} \psi+k_{y}^{2} \psi=0
$$

The governing equation for the inner layer is,

$$
\mathcal{D}^{2} \psi+K^{2} \psi-\mathrm{i} R e\left[k_{x} \frac{U_{\infty}}{\delta}-k_{z} \frac{W_{0}}{d}\right] Y_{\mathrm{s}} \psi=0
$$

where

$$
K^{2}=k_{y}^{2}\left(1-\mathrm{i} \mathcal{R}\left(1-\frac{d}{\delta}\right)\right) .
$$

With $\mathcal{R} \ll 1$, the expression for $K$ is further simplified, $K^{2} \approx k_{y}^{2}$. The asymptotic analyses presented below consider the convective-sheltered and diffusive limits of the governing equation for the inner layer. 


\section{A. Inner Layer Convective-Sheltered Regime}

The convective regime of the inner layer describes the additional sheltering caused by the transverse shear. Equation (33) can be written as

$$
\varepsilon_{\mathrm{sc}} \mathcal{D}^{2} \psi+\varepsilon_{\mathrm{sc}} k_{y}^{2} \psi-k_{y}^{2} \frac{\mathrm{i} Y_{\mathrm{s}}}{d} \psi=0
$$

where $\varepsilon_{\mathrm{sc}}=\frac{k_{y}^{2}}{R e\left(k_{x} \frac{U_{\infty}}{\delta}-k_{z} \frac{W_{0}}{d}\right) d} \ll 1$. A series expansion

$$
\psi=\psi_{0}+\varepsilon_{\mathrm{sc}} \psi_{1}+\varepsilon_{\mathrm{sc}}^{2} \psi_{2}+\ldots
$$

yields a degenerate one-term leading order equation with trivial solutions for all $\psi_{n}$, so that $\psi \equiv 0$. The boundary conditions for $\psi$ at the edge of the inner layer and at the wall require a separate treatment, which results in an edge-layer and a wall-layer with respective thicknesses,

$$
\delta_{\mathrm{se}}=\left(\frac{1 / R e}{k_{x} \frac{U_{\infty}}{\delta}-k_{z} \frac{W_{0}}{d}}\right)^{1 / 3} \quad \text { and } \quad \delta_{\mathrm{sw}}=\left(\frac{1 / R e}{d\left(k_{x} \frac{U_{\infty}}{\delta}-k_{z} \frac{W_{0}}{d}\right)}\right)^{1 / 2} .
$$

The details of the derivation are mathematically similar to those of Zaki and Saha [9] and are not repeated here. We note, however, that these layers dictate the shape of the vorticity eigenfunction near at the edge of the Stokes layer and at the wall. At both locations, viscosity thickens $\delta_{\mathrm{se}}$ and $\delta_{\mathrm{sw}}$ as inferred from their numerators. However, the thickness is also dependent on the orientation of the vorticity mode with respect to the mean shear, as described by the denominator, $k_{x} \frac{U_{\infty}}{\delta}-k_{z} \frac{W_{0}}{d}$.

The final expression for the wall-normal velocity eigenfunction is,

$$
\begin{aligned}
v= & C_{5} \exp \left(-\kappa Y_{\mathrm{s}}\right)+C_{6} \exp \left(\kappa Y_{\mathrm{s}}\right)+C_{\mathrm{sw} 2} \exp \left(-\frac{1-\mathrm{i}}{\sqrt{2} \delta_{\mathrm{w}}}\right) \\
& +C_{\mathrm{se} 1}\left[\exp \left(-\kappa Y_{\mathrm{s}}\right) \int_{-\infty}^{Y_{\mathrm{s}}} \exp (\kappa s) \varsigma(s) \mathrm{d} s\right. \\
& \left.+\exp \left(\kappa Y_{\mathrm{s}}\right) \int_{Y_{\mathrm{s}}}^{0} \exp (-\kappa s) \varsigma(s) \mathrm{d} s\right]
\end{aligned}
$$

where $\varsigma(s)=\operatorname{Ai}\left(\exp (5 \mathrm{i} \pi / 6)\left(k_{x} \frac{R e U_{\infty}}{\delta}-k_{z} \frac{R e W_{0}}{d}\right)^{1 / 3} s\right)$. The coefficients are obtained using the matching conditions of the velocities and stresses between the shear region and the free stream, and are given in the Appendix (B 1). 
Modes which belong to the inner layer convective-sheltered regime correspond to $\varepsilon_{\text {sc }} \ll 1$. The two-dimensional shear consequently introduces a dependence of the sheltering characteristics on the spanwise wavenumber $k_{z}$. Modes with high $k_{z}$ are subject to strong sheltering, as was predicted by the numerical results shown in figure 7 . The mode shape obtained from evaluating equation (35) for a wavenumber vector $\left(k_{x}, k_{y}, k_{z}\right)=(0.01, \pi, 100)$ is shown in figure 14. The modal amplitude rapidly attenuates at the beginning of the inner layer, at $d=0.5$.

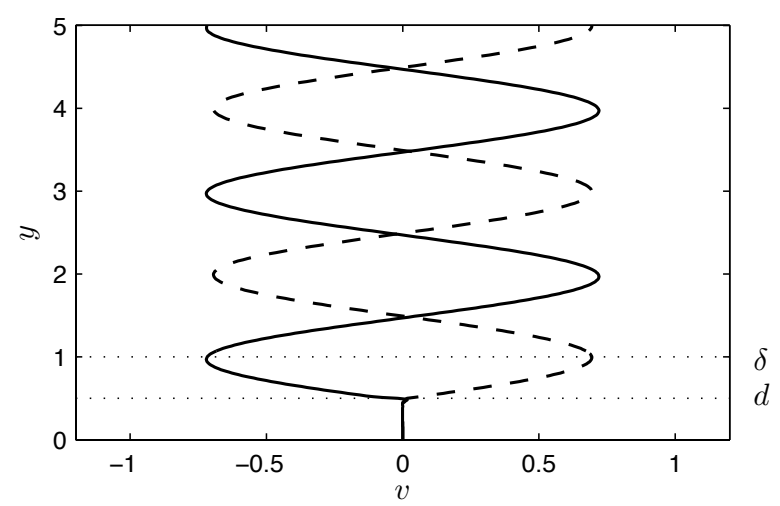

FIG. 14: Convective-sheltered modal behavior; _— , real component; - - -, imaginary component

\section{B. Inner Layer Diffusive Regime}

The diffusive regime corresponds to disturbances which retain their free-stream amplitude over the largest part of the inner layer. Free-stream modes which belong to this regime are hence scarcely impacted by the sheltering properties of the shear. Similar to the previous section, a small parameter $\varepsilon_{\mathrm{sd}}$ is introduced,

$$
\varepsilon_{\mathrm{sd}}=\frac{\operatorname{Re}\left(k_{x} \frac{U_{\infty}}{\delta}-k_{z} \frac{W_{0}}{d}\right) d}{k_{y}^{2}} \ll 1,
$$

so that the vorticity equation can be written,

$$
\frac{1}{k_{y}^{2}} \mathcal{D}^{2} \psi+\psi-\varepsilon_{\mathrm{sd}} \frac{\mathrm{i} Y_{\mathrm{s}}}{d} \psi=0
$$

A power series expansion of $\psi$ in the small parameter $\varepsilon_{\text {sd }}$ yields the leading order equation,

$$
\mathcal{D}^{2} \psi+k_{y}^{2} \psi=0
$$


whose solution is given by,

$$
\psi=C_{\mathrm{sd} 1} \exp \left(-\mathrm{i} k_{y} Y_{\mathrm{s}}\right)+C_{\mathrm{sd} 2} \exp \left(\mathrm{i} k_{y} Y_{\mathrm{s}}\right)
$$

The wall-normal velocity is therefore,

$$
\begin{aligned}
v= & C_{5} \exp \left(-\mathrm{i} k_{y} Y_{\mathrm{s}}\right)+C_{6} \exp \left(\mathrm{i} k_{y} Y_{\mathrm{s}}\right) \\
& +C_{7} \exp \left(-\kappa Y_{\mathrm{s}}\right)+C_{8} \exp \left(\kappa Y_{\mathrm{s}}\right) .
\end{aligned}
$$

The solution shows the eigenfunctions to remain oscillatory deep into the inner layer, and the coefficients are determined from the boundary conditions (see Appendix B 2).

Two classes of modes fulfil the constraint $\varepsilon_{\text {sd }} \ll 1$, and hence belong to the diffusive regime:

$$
\begin{aligned}
& \text { (i) } k_{x} \ll 1 \text { and } k_{z} \ll 1 \text {, or } \\
& \text { (ii) } \frac{k_{x}}{k_{z}} \approx \frac{W_{0} / d}{U_{\infty} / \delta}, k_{x} \text { and } k_{z} \text { of arbitrary order. }
\end{aligned}
$$

The first criterion identifies disturbances with small streamwise and spanwise wavenumbers, which permeate the shear independent of their orientation relative to the mean flow. This finding is similar to the known result of low- $k_{x}$ modes being unaffected by the streamwise shear of Blasius layers. An example eigenfunction obtained from the asymptotic solution is illustrated in figure 15, by evaluating expression (38) for a wavenumber vector $\left(k_{x}, k_{y}, k_{z}\right)=(0.1, \pi, 0.1)$. The penetration of low $\left(k_{x}, k_{z}\right)$ modes is also consistent with the results shown in figure 7 , where a region of very weak sheltering is observed at values of $k_{x} \lesssim 10^{-2}$ and $k_{z} \lesssim 10^{-1}$

When considering the second criterion, it should be emphasized that the analysis has been performed in a frame translating with $U_{\infty}$. This speed is identical to the phase speed of the continuous modes, so that one may equivalently think of the coordinate system affixed to the disturbance. Equation (40) demonstrates that, within this moving frame, modes can penetrate the boundary layer if the wall-parallel projection of their wavenumber vector, $\left(k_{x}, k_{z}\right)$ is orthogonal to the direction of the mean shear $\left(U_{\infty} / \delta, W_{0} / d\right)$. An equivalent interpretation is that phase fronts are aligned with the direction of mean shear (and not the direction of the mean flow). With this alignment, the apparent frequency for an observer within the shear is minimal (see $\S \mathrm{III}$ C), and hence penetration of the mode is maximized. An example where a mode with relatively high $\left(k_{x}, k_{z}\right)$ penetrates the inner layer due to criterion (ii) is shown in 


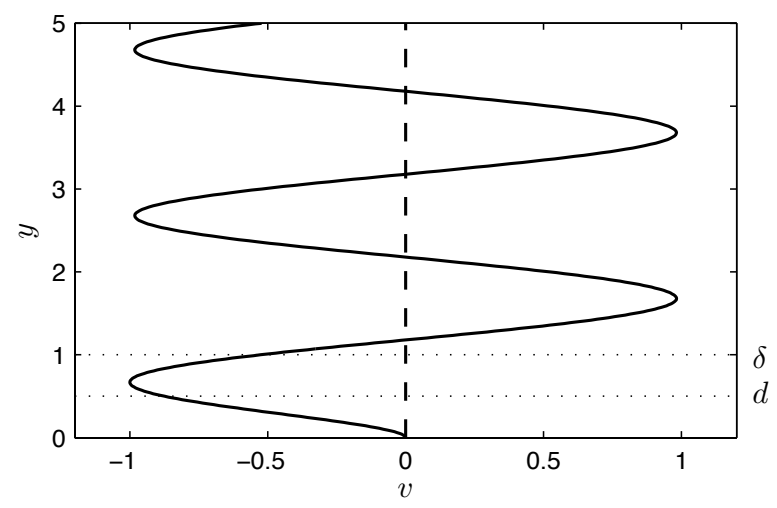

FIG. 15: Diffusive modal behavior of criterion (i); ——, real component; - - - , imaginary component

figure 16. The wavenumber vector of the eigenfunction is $\underline{k}=(1,5.5, \pi)^{T}$. Similar behavior is illustrated by the earlier Floquet results (figure 7), where a region of weak sheltering is observed around values of $k_{x} \approx 10^{0}, k_{z} \approx 10^{1}$.

Within the inner layer, eigenfunctions of criteria i and ii are both governed by the diffusion equation (37). All these modes can therefore effectively permeate the shear of the twodimensional mean flow $y \leq d$. On the other hand, modal permeability in the outer layer, $\delta>y>d$, dependens on the parameter $\mathcal{R}$. Modes that satisfy criterion (ii) may correspond to large values $\mathcal{R} \gtrsim \mathcal{O}(1)$. These modes can thus experience an attenuation in the outer layer, so that their amplitude at the edge of the inner layer is reduced from the free-stream value. This behavior is illustrated in figure 16. The integral sheltering effect for the whole boundary layer, $y \leq \delta$, may consequently be stronger for modes of criterion (ii) than it is for modes of criterion (i).

\section{CONCLUSIONS}

This work presented a method for the computation of continuous Orr-Sommerfeld eigenfunctions in the context of unsteady base flows. Periodicity of the base state results in harmonic components in the eigensolutions, which were computed using a finite order Floquet expansion in time. By considering the free-stream behavior of the continuous modes, the dispersion relation is known analytically. Using the dispersion relation, and a boundedness condition applied within the context of the Floquet expansion, the generalized eigenvalue 


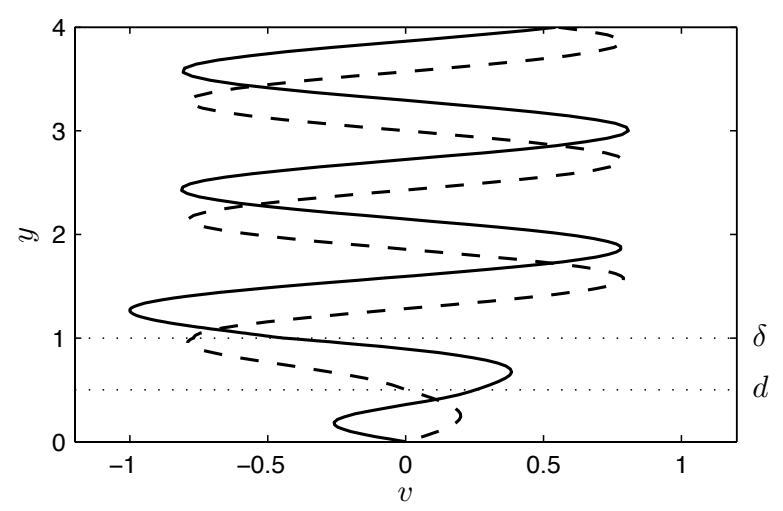

FIG. 16: Diffusive inner layer modal behavior corresponding to criterion (ii); —— , real component; - - - , imaginary component

problem of the disturbance waves is converted into a boundary value problem.

In the context of combined Stokes-Blasius flow, modal sheltering was quantified over a wide range of streamwise and spanwise wavenumbers. A substantial reduction of freestream disturbance energy entering the boundary layer in presence of unsteady shear was demonstrated. The orientation of modes subject to weak sheltering increasingly deviates from the streamwise direction for increased period and amplitude of oscillation of the base flow. The physical mechanism was explained by comparing the convective and diffusive time scales of the free-stream disturbance relative to an observer within the shear.

Asymptotic analyses of the Orr-Sommerfeld equation were performed for a twodimensional, piecewise-linear velocity profile. It was demonstrated that the effectiveness of sheltering depends on the orientation of the disturbance wavenumber vector with respect to the mean shear, as observed in a reference frame translating with the free stream: Perturbations with phase fronts aligned parallel to the mean shear are not sheltered. It was moreover shown that the outer shear permits the penetration of low $k_{x}$ modes, and the entire spectrum of $k_{z}$. On the other hand, the inner flow reorients the shear vector, and therefore introduces a new restriction on the set of penetrating modes. This further limits the set of weakly sheltered modes to the sub-spectra $\left(k_{x}, k_{z}\right) \approx(0,0)$ and $\left(k_{x}, k_{z}\right) \approx\left(\tau_{z}, \tau_{x}\right)$, where $\tau_{(x, z)}$ is the shear due to the streamwise and spanwise mean velocities, respectively. Hence, the wavenumber sets of penetrating modes for outer and inner layer are not identical, and their common elements constitute the set of modes that retain their amplitude throughout 
the full boundary layer.

In the case of continuously varying shear direction, the filtering criterion continuously changes inside the boundary layer, thus effectively limiting the disturbances which penetrate from the free stream towards the wall: The sub-spectrum that penetrates the outer layers is not necessarily effective at penetrating the inner regions of the shear. A final verdict on the role of time-periodic shear in the context of subcritical breakdown to turbulence must take into account the characteristics of sheltering. However, the boundary layer response to penetrating disturbances $[17,22]$ must also be considered, as well as the threshold for secondary instability that precedes breakdown to turbulence [21]. The current study nevertheless clearly demonstrates that the addition of spanwise time-harmonic motion appreciably reduces the permeability of energy from the free stream into the boundary layer.

\section{Acknowledgements}

The authors are grateful to Dr. Nicholas J. Vaughan for his assistance with the Floquet algorithm. This work was supported by the UK Engineering and Physical Sciences Research Council (EP/F045093) and the Air Force Office of Scientific Research (FA8655-11-M-4002).

\section{Appendix A: Validity of the Analytical Solution for the Spanwise Flow}

The present work made use of the analytical solution to the second problem of Stokes (1) in order to prescribe the time-harmonic component of the base flow. The simultaneous presence of a streamwise boundary layer nevertheless introduces a one-sided coupling between the streamwise and spanwise momentum equations for the base flow. Therefore, the Stokes solution (1) is no longer exact. Thence direct computations were employed in order to quantify the deviation of our base-flow ansatz from numerical solution of the full Navier-Stokes equations at the target Reynolds number, $\operatorname{Re}_{\delta}=1000$. The root-mean-square of the relative difference was evaluated,

$$
W_{\text {diff, rms }}(y)=\frac{1}{T W_{0}} \sqrt{\int_{0}^{T}\left(W_{\text {comp }}(y, t)-W_{\text {Stokes }}(y, t)\right)^{2} \mathrm{~d} t}
$$

and is plotted in figure 17 as a function of the wall distance for two representative periods of oscillation of the base flow. For the base-flow parameters of interest, one may thus 
conclude that the analytical solution of the second problem of Stokes (1) provides an accurate representation of the actual flow field.

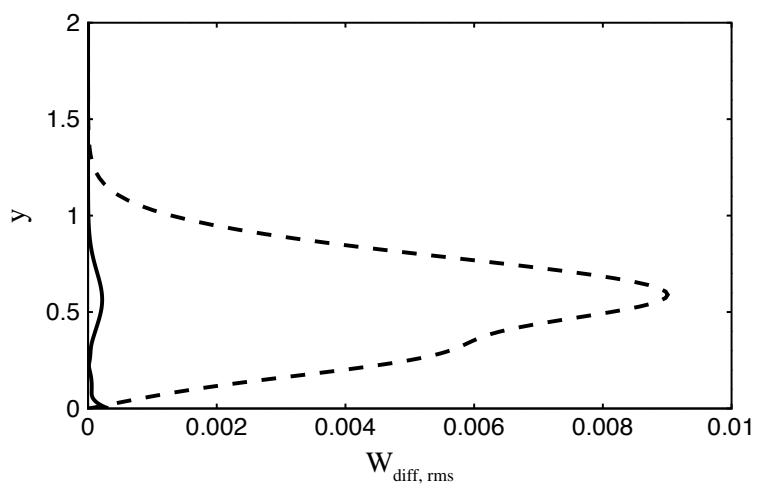

FIG. 17: Relative deviation of analytical and numerical solution for the spanwise mean flow component at the target Reynolds number; —, $\mathrm{T}=40 ;---, \mathrm{T}=400$

\section{Appendix B: Coefficients of the Asymptotic Solutions}

The below given coefficients follow from the application of the boundary conditions,

$$
\begin{gathered}
v(y=0)=0 \\
\mathcal{D} v(y=0)=0 \\
v(y \rightarrow \infty)<\infty \\
\mathcal{D} v(y \rightarrow \infty)<\infty .
\end{gathered}
$$

Conditions (B3) and (B4) correspond to the boundedness of modal amplitude and wallnormal derivative in the limit of large wall distances mentioned in the discussion of the continuous spectrum, cf. section II B.

Additionally, four interface conditions are required for matching the outer and inner layer 
solutions at $Y_{\mathrm{s}}=0$,

$$
\begin{aligned}
v\left(Y_{\mathrm{s}}=0^{+}\right)-v\left(Y_{\mathrm{s}}=0^{-}\right) & =0 \\
\mathcal{D} v\left(Y_{\mathrm{s}}=0^{+}\right)-\mathcal{D} v\left(Y_{\mathrm{s}}=0^{-}\right) & =\frac{k_{z} W_{0} v\left(Y_{\mathrm{s}}=0\right)}{d\left(\omega-k_{z} W\left(Y_{\mathrm{s}}=0\right)\right)} \\
\mathcal{D}^{2} v\left(Y_{\mathrm{s}}=0^{+}\right)-\mathcal{D}^{2} v\left(Y_{\mathrm{s}}=0^{-}\right) & =0 \\
\mathcal{D}^{3} v\left(Y_{\mathrm{s}}=0^{+}\right)-\mathcal{D}^{3} v\left(Y_{\mathrm{s}}=0^{-}\right) & =3 \kappa^{2} \frac{k_{z} W_{0} v\left(Y_{\mathrm{s}}=0\right)}{d\left(\omega-k_{z} W\left(Y_{\mathrm{s}}=0\right)\right)} .
\end{aligned}
$$

\section{Convective Inner Layer Regime}

The coefficients are

$$
\begin{aligned}
C_{\mathrm{se} 2}= & \lambda C_{\mathrm{se} 1} \\
C_{\mathrm{se} 1}= & C_{1}\left[\frac{\mathrm{Ai}(0)}{\kappa^{2}+k_{y}^{2}}+A^{-}-A^{+}-\lambda\left(\frac{k_{y}^{2}+\zeta^{2}}{\kappa^{2}+k_{y}^{2}} \exp (\zeta d)+\frac{\zeta-\kappa}{2 \kappa} \exp (-\kappa \delta)+\frac{\kappa+\zeta}{2 \kappa} \exp (\kappa d)\right)\right]^{-1} \\
C_{1}= & \frac{1}{2}\left[-C_{\mathrm{se} 1} \frac{\mathrm{Ai}(\varsigma(0))+\mathrm{Ai}^{\prime}(\varsigma(0)) \frac{\varsigma^{\prime}}{\mathrm{i} k_{y}}}{\kappa^{2}+k_{y}^{2}}+C_{\mathrm{sw} 2}\left(1+\frac{\zeta}{\mathrm{i} k_{y}}\right) \frac{\kappa^{2}-\zeta^{2}}{\kappa^{2}+\zeta^{2}} \exp (\zeta d)\right. \\
& \left.-\frac{2 \operatorname{Re} \kappa^{2}\left(k_{x} U_{\infty} / \delta-k_{z} W_{0} / d\right)}{k_{y}\left(\kappa^{2}+k_{y}^{2}\right)^{2}}\left(C_{\mathrm{sw} 2} \frac{\kappa^{2}-\zeta^{2}}{\kappa^{2}+\zeta^{2}} \exp (\zeta d)-C_{\mathrm{se} 1} \frac{\mathrm{Ai}(0)}{\kappa^{2}+k_{y}^{2}}+C_{3}\right)\right] \\
C_{2}= & \frac{\mathrm{Ai}(\varsigma(0))-\mathrm{Ai}^{\prime}(\varsigma(0)) \frac{\varsigma^{\prime}}{\mathrm{i} k_{y}}}{\kappa^{2}+k_{y}^{2}}+C_{\mathrm{sw} 2}\left(1+\frac{\zeta}{\mathrm{i} k_{y}}\right) \frac{\kappa^{2}-\zeta^{2}}{\kappa^{2}+\zeta^{2}} \exp (\zeta d) \\
& \left.+\frac{2 \operatorname{Re} \kappa^{2}\left(k_{x} U_{\infty} / \delta-k_{z} W_{0} / d\right)}{k_{y}\left(\kappa^{2}+k_{y}^{2}\right)^{2}}\left(C_{\mathrm{sw} 2} \frac{\kappa^{2}-\zeta^{2}}{\kappa^{2}+\zeta^{2}} \exp (\zeta d)-C_{\mathrm{se} 1} \frac{\mathrm{Ai}(0)}{\kappa^{2}+k_{y}^{2}}+C_{3}\right)\right] \\
C_{5}= & -\frac{\kappa+\zeta}{2 \kappa} C_{\mathrm{sw} 2} \exp (\kappa d) \\
C_{6}= & -C_{\mathrm{se} 1} A^{+}-\frac{\kappa-\zeta}{2 \kappa} C_{\mathrm{sw} 2} \exp (-\kappa d)
\end{aligned}
$$

with

$$
A^{ \pm}=\frac{1}{2 \kappa} \int_{-d}^{0} \exp ( \pm \kappa s) \operatorname{Ai}(\varsigma(s)) \mathrm{d} s
$$


and

$$
\lambda=\frac{\frac{1}{\kappa^{2}+k_{y}^{2}}\left(\kappa \operatorname{Ai}(0)-\operatorname{Ai}^{\prime}(\varsigma(0)) \varsigma^{\prime}(0)\right)-2 \kappa A^{+}-\frac{\mathrm{i} R e\left(k_{x} U_{\infty} / \delta-k_{z} W_{0} / d\right)\left(3 \kappa^{2}+k_{y}^{2}\right)}{\left(\kappa^{2}+k_{y}^{2}\right)^{2}}\left(A^{-}-A^{+}\right)}{(\kappa-\zeta)\left(\exp (-\kappa d)-\frac{k_{y}^{2}+\zeta^{2}}{\kappa^{2}+k_{y}^{2}} \exp (\zeta d)\right)+\frac{\mathrm{i} \operatorname{Re}\left(k_{x} U_{\infty} / \delta-k_{z} W_{0} / d\right)}{\left(\kappa^{2}+k_{y}^{2}\right)^{2}}\left(\exp (\zeta d)+\frac{\zeta-\kappa}{2 \kappa} \exp (-\kappa d)\right)},
$$

and $\varsigma\left(Y_{\mathrm{s}}\right)=\exp (5 \mathrm{i} \pi / 6)\left(R e\left(k_{x} U_{\infty} / \delta-k_{z} W_{0} / d\right)\right)^{1 / 3} Y_{\mathrm{s}}$ and $\zeta=(1-\mathrm{i}) /\left(\sqrt{2} \delta_{\mathrm{sw}}\right)$.

\section{Diffusive Inner Layer Regime}

The coefficients are

$$
\begin{aligned}
& C_{5}=\frac{2 C_{3} \exp \left(-\mathrm{i} k_{y} d\right)}{\left(\left(-1+\frac{\mathrm{i} k_{y}}{\kappa}\right)+\lambda\left(1+\frac{\mathrm{i} k_{y}}{\kappa}\right)\right) \exp (\kappa d)+\left(-\left(1+\frac{\mathrm{i} k_{y}}{\kappa}\right)+\lambda\left(1-\frac{\mathrm{i} k_{y}}{\kappa}\right)\right) \exp (-\kappa d)} \\
& C_{6}=-\lambda C_{5} \exp \left(2 \mathrm{i} k_{y} d\right) \\
& C_{1}=C_{5}+\frac{R e \kappa^{2}\left(k_{x} U_{\infty} / \delta-k_{z} W_{0} / d\right)}{k_{y}\left(\kappa^{2}+k_{y}^{2}\right)^{2}}\left(C_{3}+C_{5}+C_{6}\right) \\
& C_{2}=C_{6}-\frac{R e \kappa^{2}\left(k_{x} U_{\infty} / \delta-k_{z} W_{0} / d\right)}{k_{y}\left(\kappa^{2}+k_{y}^{2}\right)^{2}}\left(C_{3}+C_{5}+C_{6}\right) \\
& C_{7}=-\frac{\exp (-\kappa d)}{2}\left(\left(1+\frac{\mathrm{i} k_{y}}{\kappa}\right) C_{5} \exp \left(\mathrm{i} k_{y} d\right)+\left(1-\frac{\mathrm{i} k_{y}}{\kappa}\right) C_{6} \exp \left(-\mathrm{i} k_{y} d\right)\right) \\
& C_{8}=-\frac{\exp (\kappa d)}{2}\left(\left(-1+\frac{\mathrm{i} k_{y}}{\kappa}\right) C_{5} \exp \left(\mathrm{i} k_{y} d\right)-\left(1-\frac{\mathrm{i} k_{y}}{\kappa}\right) C_{6} \exp \left(-\mathrm{i} k_{y} d\right)\right)
\end{aligned}
$$

with

$$
\lambda=\frac{(2 \kappa+\gamma)\left(-1+\frac{\mathrm{i} k_{y}}{\kappa}\right) \exp (\kappa d)+\gamma}{(-2 \kappa+\gamma)\left(1+\frac{\mathrm{i} k_{y}}{\kappa}\right) \exp (\kappa d)+\gamma},
$$

where $\gamma=\mathrm{i} R e\left(k_{x} U_{\infty} / \delta-k_{z} W_{0} / d\right) /\left(\kappa^{2}+k_{y}^{2}\right)^{2}$.

[1] R. Jordinson, Spectrum of eigenvalues of the Orr-Sommerfeld equation for Blasius flow, Physics of Fluids 14, 2535 (1971).

[2] L. M. Mack, A numerical study of the temporal eigenvalue spectrum of the Blasius boundary layer, Journal of Fluid Mechanics 73, 497 (1976). 
[3] C. E. Grosch and H. Salwen, The continuous spectrum of the Orr-Sommerfeld equation. Part 1. The spectrum and the eigenfunctions, Journal of Fluid Mechanics 87, 33 (1978).

[4] R. G. Jacobs and P. A. Durbin, Shear sheltering and the continuous spectrum of the OrrSommerfeld Equation, Physics of Fluids 10, 2006 (1998).

[5] J. C. R. Hunt, A review of rapidly distorted turbulent flows and its applications, Fluid Dynamics Transactions 9, 121 (1977).

[6] J. C. R. Hunt and D. J. Carruthers, Rapid distortion theory and the 'problems' of turbulence, Journal of Fluid Mechanics 212, 497 (1990).

[7] A. D. D. Craik, The continuous spectrum of the Orr-Sommerfeld equation: Note on a paper of Grosch and Salwen, Journal of Fluid Mechanics 226, 565 (1991).

[8] J. C. R. Hunt and P. A. Durbin, Perturbed vortical layers and shear sheltering, Fluid Dynamics Research 464 (1999).

[9] T. A. Zaki and S. Saha, On shear sheltering and the structure of vortical modes in single- and two-fluid boundary layers, Journal of Fluid Mechanics 626, 111 (2009).

[10] L.-U. Schrader, L. Brandt, and D. S. Henningson, Receptivity mechanisms in three-dimensional boundary-layer flows, Journal of Fluid Mechanics 618, 209 (2009).

[11] R. G. Jacobs and P. A. Durbin, Simulations of bypass transition, Journal of Fluid Mechanics 428, 185 (2001).

[12] L. Brandt, P. Schlatter, and D. S. Hennginson, Transition in boundary layers subject to freestream turbulence, Journal of Fluid Mechanics 517, 167 (2004).

[13] T. A. Zaki and P. A. Durbin, Mode interaction and the bypass route to transition, Journal of Fluid Mechanics 531, 85 (2005).

[14] P. Moin, T.-H. Shih, D. Driver, and N. N. Mansour, Direct numerical simulation of a threedimensional turbulent boundary layer, Physics of Fluids 2, 1846 (1990).

[15] W. J. Jung, N. Mangiavacchi, and R. Akhavan, Suppression of turbulence in wall-bounded flows by high-frequency spanwise oscillations, Physics of Fluids 4, 1605 (1992).

[16] G. E. Karniadakis and K. W. Choi, Mechanisms on transverse motions in turbulent wall flows, Annual Review of Fluid Mechanics 35, 45 (2003).

[17] P. Ricco, Laminar streaks with spanwise wall forcing, Physics of Fluids 23 (2011).

[18] P. Hall, The linear stability of flat Stokes layers, Proceedings of the Royal Society A 359, 151 (1978). 
[19] T. Herbert, Secondary instability of boundary layers, Annual Review of Fluid Mechanics 20, 487 (1988).

[20] Y. Liu, T. A. Zaki, and P. A. Durbin, Floquet analysis of secondary instability of boundary layers distorted by Klebanoff streaks and Tollmien-Schlichting waves, Physics of Fluids 20, $124102(2008)$.

[21] N. J. Vaughan and T. A. Zaki, Stability of zero-pressure-gradient boundary layer distorted by unsteady Klebanoff streaks, Journal of Fluid Mechanics 681, 116-153 (2011).

[22] P. Andersson, M. Bergren, and D. S. Henningson, Optimal disturbances and bypass transition in boundary layers, Physics of Fluids 11, 134 (1999).

[23] T. A. Zaki and P. A. Durbin, Continuous mode transition and the effects of pressure gradients, Journal of Fluid Mechanics 563, 357 (2006).

[24] T. Von Kármán, Progress in the statistical theory of turbulence, Proceedings of the National Academy of Sciences 34, 530 (1948).

[25] R. S. Rogallo, Numerical experiments in homogeneous turbulence, National Aeronautics and Space Administration (NASA) Tech Report TM-81315, (1981). 